\title{
A note on modular forms and generalized anomaly cancellation formulas
}

\author{
Kefeng Liu and Yong Wang
}

\begin{abstract}
By studying modular invariance properties of some characteristic forms, we prove some new anomaly cancellation formulas which generalize the Han-Zhang and Han-Liu-Zhang anomaly cancellation formulas
\end{abstract}

Keywords: Modular invariance; Anomaly cancellation formulas;

\section{Introduction}

In 1983, the physicists Alvarez-Gaumé and Witten [AW] discovered the "miraculous cancellation" formula for gravitational anomaly which reveals a beautiful relation between the top components of the Hirzebruch $\widehat{L}$-form and $\widehat{A}$-form of a 12-dimensional smooth Riemannian manifold. Kefeng Liu [L] established higher dimensional "miraculous cancellation" formulas for $(8 k+4)$-dimensional Riemannian manifolds by developing modular invariance properties of characteristic forms. These formulas could be used to deduce some divisibility results. In [HZ1], [HZ2], for each $(8 k+4)$-dimensional smooth Riemannian manifold, a more general cancellation formula that involves a complex line bundle was established. This formula was applied to $\operatorname{spin}^{c}$ manifolds, then an analytic Ochanine congruence formula was derived. In [CH1], Qingtao Chen and Fei Han obtained more twisted cancellation formulas for $8 k$ and $8 k+4$ dimensional manifolds and they also applied their cancellation formulas to study divisibilities on spin manifolds and congruences on $\operatorname{spin}^{c}$ manifolds. Recently, Han, Liu and Zhang generalized the anomaly cancellation formulas to cases that an auxiliary bundle as well as a complex line bundle are involved with non conditions on the first Pontryagin forms being assumed in [HLZ]. On the other hand, motivated by the Chern-Simons theory, in [CH2], Qingtao Chen and Fei Han computed the transgressed forms of some modularly invariant characteristic forms, which are related to the elliptic genera. They studied the modularity properties of these secondary characteristic forms and relations among them. In [W], Wang generalized the Chen-Han cancellation formulas to the case that a complex line bundle is involved. One naturally asks if there exist more cancellation formulas similar to the Han-Liu-Zhang cancellation formulas. In this note, we derive more these type cancellation formulas.

This paper is organized as follows: In Section 2, we review some knowledge on characteristic forms and modular forms that we are going to use. In Section 3, we 
generalize the Han-Liu-Zhang cancellation formulas to the (a,b) type cancellation formulas. In Section 4, we prove some general cancellation formulas involving two complex line bundles. In Section 5, we study modular invariance properties of some characteristic forms on odd dimensional manifolds.

\section{2 characteristic forms and modular forms}

The purpose of this section is to review the necessary knowledge on characteristic forms and modular forms that we are going to use.

2.1 characteristic forms. Let $M$ be a Riemannian manifold. Let $\nabla^{T M}$ be the associated Levi-Civita connection on $T M$ and $R^{T M}=\left(\nabla^{T M}\right)^{2}$ be the curvature of $\nabla^{T M}$. Let $\widehat{A}\left(T M, \nabla^{T M}\right)$ and $\widehat{L}\left(T M, \nabla^{T M}\right)$ be the Hirzebruch characteristic forms defined respectively by (cf. [Z])

$$
\begin{aligned}
& \widehat{A}\left(T M, \nabla^{T M}\right)=\operatorname{det}^{\frac{1}{2}}\left(\frac{\frac{\sqrt{-1}}{4 \pi} R^{T M}}{\sinh \left(\frac{\sqrt{-1}}{4 \pi} R^{T M}\right)}\right), \\
& \widehat{L}\left(T M, \nabla^{T M}\right)=\operatorname{det}^{\frac{1}{2}}\left(\frac{\frac{\sqrt{-1}}{2 \pi} R^{T M}}{\tanh \left(\frac{\sqrt{-1}}{4 \pi} R^{T M}\right)}\right) .
\end{aligned}
$$

Let $E, F$ be two Hermitian vector bundles over $M$ carrying Hermitian connection $\nabla^{E}, \nabla^{F}$ respectively. Let $R^{E}=\left(\nabla^{E}\right)^{2}$ (resp. $R^{F}=\left(\nabla^{F}\right)^{2}$ ) be the curvature of $\nabla^{E}$ (resp. $\nabla^{F}$ ). If we set the formal difference $G=E-F$, then $G$ carries an induced Hermitian connection $\nabla^{G}$ in an obvious sense. We define the associated Chern character form as

$$
\operatorname{ch}\left(G, \nabla^{G}\right)=\operatorname{tr}\left[\exp \left(\frac{\sqrt{-1}}{2 \pi} R^{E}\right)\right]-\operatorname{tr}\left[\exp \left(\frac{\sqrt{-1}}{2 \pi} R^{F}\right)\right] .
$$

For any complex number $t$, let

$$
\wedge_{t}(E)=\left.\mathbf{C}\right|_{M}+t E+t^{2} \wedge^{2}(E)+\cdots, S_{t}(E)=\left.\mathbf{C}\right|_{M}+t E+t^{2} S^{2}(E)+\cdots
$$

denote respectively the total exterior and symmetric powers of $E$, which live in $K(M)[[t]]$. The following relations between these operations hold,

$$
S_{t}(E)=\frac{1}{\wedge_{-t}(E)}, \wedge_{t}(E-F)=\frac{\wedge_{t}(E)}{\bigwedge_{t}(F)} .
$$

Moreover, if $\left\{\omega_{i}\right\},\left\{\omega_{j}^{\prime}\right\}$ are formal Chern roots for Hermitian vector bundles $E, F$ respectively, then

$$
\operatorname{ch}\left(\wedge_{t}(E)\right)=\prod_{i}\left(1+e^{\omega_{i}} t\right) .
$$


Then we have the following formulas for Chern character forms,

$$
\operatorname{ch}\left(S_{t}(E)\right)=\frac{1}{\prod_{i}\left(1-e^{\omega_{i}} t\right)}, \operatorname{ch}\left(\wedge_{t}(E-F)\right)=\frac{\prod_{i}\left(1+e^{\omega_{i}} t\right)}{\prod_{j}\left(1+e^{\omega_{j}^{\prime}} t\right)} .
$$

If $W$ is a real Euclidean vector bundle over $M$ carrying a Euclidean connection $\nabla^{W}$, then its complexification $W_{\mathbf{C}}=W \otimes \mathbf{C}$ is a complex vector bundle over $M$ carrying a canonical induced Hermitian metric from that of $W$, as well as a Hermitian connection $\nabla^{W_{\mathbf{C}}}$ induced from $\nabla^{W}$. If $E$ is a vector bundle (complex or real) over $M$, set $\widetilde{E}=E-\operatorname{dim} E$ in $K(M)$ or $K O(M)$.

2.2 Some properties about the Jacobi theta functions and modular forms

We first recall the four Jacobi theta functions are defined as follows( cf. [C]):

$$
\begin{gathered}
\theta(v, \tau)=2 q^{\frac{1}{8}} \sin (\pi v) \prod_{j=1}^{\infty}\left[\left(1-q^{j}\right)\left(1-e^{2 \pi \sqrt{-1} v} q^{j}\right)\left(1-e^{-2 \pi \sqrt{-1} v} q^{j}\right)\right] \\
\theta_{1}(v, \tau)=2 q^{\frac{1}{8}} \cos (\pi v) \prod_{j=1}^{\infty}\left[\left(1-q^{j}\right)\left(1+e^{2 \pi \sqrt{-1} v} q^{j}\right)\left(1+e^{-2 \pi \sqrt{-1} v} q^{j}\right)\right], \\
\theta_{2}(v, \tau)=\prod_{j=1}^{\infty}\left[\left(1-q^{j}\right)\left(1-e^{2 \pi \sqrt{-1} v} q^{j-\frac{1}{2}}\right)\left(1-e^{-2 \pi \sqrt{-1} v} q^{j-\frac{1}{2}}\right)\right], \\
\theta_{3}(v, \tau)=\prod_{j=1}^{\infty}\left[\left(1-q^{j}\right)\left(1+e^{2 \pi \sqrt{-1} v} q^{j-\frac{1}{2}}\right)\left(1+e^{-2 \pi \sqrt{-1} v} q^{j-\frac{1}{2}}\right)\right],
\end{gathered}
$$

where $q=e^{2 \pi \sqrt{-1} \tau}$ with $\tau \in \mathbf{H}$, the upper half complex plane. Let

$$
\theta^{\prime}(0, \tau)=\left.\frac{\partial \theta(v, \tau)}{\partial v}\right|_{v=0}
$$

Then the following Jacobi identity (cf. [Ch]) holds,

$$
\theta^{\prime}(0, \tau)=\pi \theta_{1}(0, \tau) \theta_{2}(0, \tau) \theta_{3}(0, \tau)
$$

Denote $S L_{2}(\mathbf{Z})=\left\{\left(\begin{array}{cc}a & b \\ c & d\end{array}\right) \mid a, b, c, d \in \mathbf{Z}, a d-b c=1\right\}$ the modular group. Let $S=\left(\begin{array}{cc}0 & -1 \\ 1 & 0\end{array}\right), T=\left(\begin{array}{cc}1 & 1 \\ 0 & 1\end{array}\right)$ be the two generators of $S L_{2}(\mathbf{Z})$. They act on $\mathbf{H}$ by $S \tau=-\frac{1}{\tau}, T \tau=\tau+1$. One has the following transformation laws of theta functions under the actions of $S$ and $T$ (cf. [C]):

$$
\begin{gathered}
\theta(v, \tau+1)=e^{\frac{\pi \sqrt{-1}}{4}} \theta(v, \tau), \quad \theta\left(v,-\frac{1}{\tau}\right)=\frac{1}{\sqrt{-1}}\left(\frac{\tau}{\sqrt{-1}}\right)^{\frac{1}{2}} e^{\pi \sqrt{-1} \tau v^{2}} \theta(\tau v, \tau) \\
\theta_{1}(v, \tau+1)=e^{\frac{\pi \sqrt{-1}}{4}} \theta_{1}(v, \tau), \quad \theta_{1}\left(v,-\frac{1}{\tau}\right)=\left(\frac{\tau}{\sqrt{-1}}\right)^{\frac{1}{2}} e^{\pi \sqrt{-1} \tau v^{2}} \theta_{2}(\tau v, \tau)
\end{gathered}
$$




$$
\begin{aligned}
& \theta_{2}(v, \tau+1)=\theta_{3}(v, \tau), \quad \theta_{2}\left(v,-\frac{1}{\tau}\right)=\left(\frac{\tau}{\sqrt{-1}}\right)^{\frac{1}{2}} e^{\pi \sqrt{-1} \tau v^{2}} \theta_{1}(\tau v, \tau) \\
& \theta_{3}(v, \tau+1)=\theta_{2}(v, \tau), \quad \theta_{3}\left(v,-\frac{1}{\tau}\right)=\left(\frac{\tau}{\sqrt{-1}}\right)^{\frac{1}{2}} e^{\pi \sqrt{-1} \tau v^{2}} \theta_{3}(\tau v, \tau), \\
& \theta^{\prime}(v, \tau+1)=e^{\frac{\pi \sqrt{-1}}{4}} \theta^{\prime}(v, \tau), \quad \theta^{\prime}\left(0,-\frac{1}{\tau}\right)=\frac{1}{\sqrt{-1}}\left(\frac{\tau}{\sqrt{-1}}\right)^{\frac{1}{2}} \tau \theta^{\prime}(0, \tau) .
\end{aligned}
$$

Definition 2.1 A modular form over $\Gamma$, a subgroup of $S L_{2}(\mathbf{Z})$, is a holomorphic function $f(\tau)$ on $\mathbf{H}$ such that

$$
f(g \tau):=f\left(\frac{a \tau+b}{c \tau+d}\right)=\chi(g)(c \tau+d)^{k} f(\tau), \quad \forall g=\left(\begin{array}{cc}
a & b \\
c & d
\end{array}\right) \in \Gamma,
$$

where $\chi: \Gamma \rightarrow \mathbf{C}^{\star}$ is a character of $\Gamma$. $k$ is called the weight of $f$.

Let

$$
\begin{aligned}
& \Gamma_{0}(2)=\left\{\left(\begin{array}{ll}
a & b \\
c & d
\end{array}\right) \in S L_{2}(\mathbf{Z}) \mid c \equiv 0(\bmod 2)\right\}, \\
& \Gamma^{0}(2)=\left\{\left(\begin{array}{ll}
a & b \\
c & d
\end{array}\right) \in S L_{2}(\mathbf{Z}) \mid b \equiv 0(\bmod 2)\right\},
\end{aligned}
$$

be the two modular subgroups of $S L_{2}(\mathbf{Z})$. It is known that the generators of $\Gamma_{0}(2)$ are $T, S T^{2} S T$, the generators of $\Gamma^{0}(2)$ are $S T S, T^{2} S T S$ (cf.[C]).

Let $E_{2}(\tau)$ be Eisenstein series which is a quasimodular form over $S L(2, \mathbf{Z})$, satisfying:

$$
E_{2}\left(\frac{a \tau+b}{c \tau+d}\right)=(c \tau+d)^{2} E_{2}(\tau)-\frac{6 \sqrt{-1} c(c \tau+d)}{\pi} .
$$

In particular, we have

$$
\begin{gathered}
E_{2}(\tau+1)=E_{2}(\tau), \\
E_{2}\left(-\frac{1}{\tau}\right)=\tau^{2} E_{2}(\tau)-\frac{6 \sqrt{-1} \tau}{\pi} .
\end{gathered}
$$

If $\Gamma$ is a modular subgroup, let $\mathcal{M}_{\mathbf{R}}(\Gamma)$ denote the ring of modular forms over $\Gamma$ with real Fourier coefficients. Writing $\theta_{j}=\theta_{j}(0, \tau), 1 \leq j \leq 3$, we introduce four explicit modular forms (cf. [L]),

$$
\begin{gathered}
\delta_{1}(\tau)=\frac{1}{8}\left(\theta_{2}^{4}+\theta_{3}^{4}\right), \quad \varepsilon_{1}(\tau)=\frac{1}{16} \theta_{2}^{4} \theta_{3}^{4}, \\
\delta_{2}(\tau)=-\frac{1}{8}\left(\theta_{1}^{4}+\theta_{3}^{4}\right), \quad \varepsilon_{2}(\tau)=\frac{1}{16} \theta_{1}^{4} \theta_{3}^{4} .
\end{gathered}
$$

They have the following Fourier expansions in $q^{\frac{1}{2}}$ :

$$
\delta_{1}(\tau)=\frac{1}{4}+6 q+\cdots, \quad \varepsilon_{1}(\tau)=\frac{1}{16}-q+\cdots,
$$




$$
\delta_{2}(\tau)=-\frac{1}{8}-3 q^{\frac{1}{2}}+\cdots, \quad \varepsilon_{2}(\tau)=q^{\frac{1}{2}}+\cdots,
$$

where the "..." terms are the higher degree terms, all of which have integral coefficients. They also satisfy the transformation laws,

$$
\delta_{2}\left(-\frac{1}{\tau}\right)=\tau^{2} \delta_{1}(\tau), \quad \varepsilon_{2}\left(-\frac{1}{\tau}\right)=\tau^{4} \varepsilon_{1}(\tau),
$$

Lemma $2.2([\mathrm{~L}]) \delta_{1}(\tau)$ (resp. $\varepsilon_{1}(\tau)$ ) is a modular form of weight 2 (resp. 4) over $\Gamma_{0}(2), \delta_{2}(\tau)$ (resp. $\varepsilon_{2}(\tau)$ ) is a modular form of weight 2 (resp. 4) over $\Gamma^{0}(2)$ and moreover $\mathcal{M}_{\mathbf{R}}\left(\Gamma^{0}(2)\right)=\mathbf{R}\left[\delta_{2}(\tau), \varepsilon_{2}(\tau)\right]$.

\section{A generalization of the Han-Liu-Zhang cancellation formulas}

Let $M$ be a $4 k$ dimensional Riemannian manifold and $V$ be a rank $2 l$ real vector bundle on $M$. Let $a, b$ be two integers. Set

$$
\begin{gathered}
\Theta_{1}\left(T_{C} M, V_{C}, a, b\right)=\bigotimes_{n=1}^{\infty} S_{q^{n}}\left(\widetilde{T_{C} M}\right) \otimes \bigotimes_{m=1}^{\infty} \wedge_{q^{m}}\left(\widetilde{V_{C}}\right)^{a} \\
\otimes\left(\bigotimes_{r=1}^{\infty} \wedge_{q^{r-\frac{1}{2}}}\left(\widetilde{V_{C}}\right)\right)^{b} \otimes\left(\bigotimes_{s=1}^{\infty} \wedge_{-q^{s-\frac{1}{2}}}\left(\widetilde{V_{C}}\right)\right)^{b}, \\
\Theta_{2}\left(T_{C} M, V_{C}, a, b\right)=\bigotimes_{n=1}^{\infty} S_{q^{n}}\left(\widetilde{T_{C} M}\right) \otimes\left(\bigotimes_{m=1}^{\infty} \wedge_{q^{m}}\left(\widetilde{V_{C}}\right)\right)^{b} \\
\otimes\left(\bigotimes_{r=1}^{\infty} \wedge_{q^{r-\frac{1}{2}}}\left(\widetilde{V_{C}}\right)\right)^{b} \otimes\left(\bigotimes_{s=1}^{\infty} \wedge_{-q^{s-\frac{1}{2}}}\left(\widetilde{V_{C}}\right)\right)^{a},
\end{gathered}
$$

Clearly, $\Theta_{1}\left(T_{C} M, V_{C}, a, b\right)$ and $\Theta_{2}\left(T_{C} M, V_{C}, a, b\right)$ admit formal Fourier expansion in $q^{\frac{1}{2}}$ as

$$
\begin{gathered}
\Theta_{1}\left(T_{C} M, V_{C}, a, b\right)=A_{0}\left(T_{C} M, V_{C}, a, b\right)+A_{1}\left(T_{C} M, V_{C}, a, b\right) q^{\frac{1}{2}}+\cdots, \\
\Theta_{2}\left(T_{C} M, V_{C}, a, b\right)=B_{0}\left(T_{C} M, V_{C}, a, b\right)+B_{1}\left(T_{C} M, V_{C}, a, b\right) q^{\frac{1}{2}}+\cdots,
\end{gathered}
$$

where the $A_{j}$ and $B_{j}$ are elements in the semi-group formally generated by Hermitian vector bundles over $M$. Moreover, they carry canonically induced Hermitian connections. Let $\left\{ \pm 2 \pi i y_{\nu}\right\}$ be the formal Chern roots of $V_{C}$. If $V$ is spin and $\triangle(V)$ is the spinor bundle of $V$, one know that the Chern character of $\triangle(V)$ is given by

$$
\operatorname{ch}(\triangle(V))=\prod_{\nu=1}^{l}\left(e^{\pi i y_{\nu}}+e^{-\pi i y_{\nu}}\right) .
$$

In the following, we do not assume that $V$ is spin, but still formally use $\operatorname{ch}\left((\triangle(V))^{a}\right)$ for the short-hand notion of $\left(\prod_{\nu=1}^{l}\left(e^{\pi i y_{\nu}}+e^{-\pi i y_{\nu}}\right)\right)^{a}$ which is a well-defined cohomology class on $M$. Let $p_{1}$ denote the first Pontryagin class. If $\omega$ is a differential form 
over $M$, we denote $\omega^{(4 k)}$ its top degree component. Define virtual complex vector bundle $b_{r}\left(T_{\mathbf{C}} M, V_{\mathbf{C}}, a, b\right)$ on $M, 0 \leq r \leq\left[\frac{k}{2}\right]$, via the equality

$$
\Theta_{2}\left(T_{C} M, V_{C}, a, b\right) \equiv \sum_{r=0}^{\left[\frac{k}{2}\right]} b_{r}\left(8 \delta_{2}\right)^{k-2 r} \varepsilon_{2}^{r} \quad \bmod q^{\frac{\left[\frac{k}{2}\right]+1}{2}} \dot{K}(M)\left[\left[q^{\frac{1}{2}}\right]\right] .
$$

Then

$$
b_{0}=(-1)^{k} \mathbf{C}, \quad b_{1}=-24(-1)^{k} k-a \widetilde{V_{\mathbf{C}}} .
$$

Define degree $4 k-4$ differential forms $\beta_{r}\left(T_{\mathbf{C}} M, V_{\mathbf{C}}, a, b\right)$ on $M, 0 \leq r \leq\left[\frac{k}{2}\right]$, via the equality

$$
\begin{gathered}
\left\{\frac{e^{\frac{1}{24} E_{2}(\tau)\left[p_{1}(T M)-(a+2 b) p_{1}(V)\right]}-1}{p_{1}(T M)-(a+2 b) p_{1}(V)} \widehat{A}(T M) \operatorname{ch}\left((\triangle(V))^{b}\right) \operatorname{ch}\left(\Theta_{2}\left(T_{C} M, V_{C}, a, b\right)\right)\right\}^{(4 k-4)} \\
\equiv \sum_{r=0}^{\left[\frac{k}{2}\right]} \beta_{r}\left(8 \delta_{2}\right)^{k-2 r} \varepsilon_{2}^{r} \quad \bmod q^{\frac{\left[\frac{k}{2}\right]+1}{2}} \dot{\Omega}^{(4 k-4)}(M)\left[\left[q^{\frac{1}{2}}\right]\right]
\end{gathered}
$$

It is easy to calculate that

$$
\begin{gathered}
\beta_{0}=(-1)^{k}\left\{\frac{e^{\frac{1}{24}\left[p_{1}(T M)-(a+2 b) p_{1}(V)\right]}-1}{p_{1}(T M)-(a+2 b) p_{1}(V)} \widehat{A}(T M) \operatorname{ch}\left((\triangle(V))^{b}\right)\right\}^{(4 k-4)}, \\
\beta_{1}=(-1)^{k}\left\{\frac{e^{\frac{1}{24}\left[p_{1}(T M)-(a+2 b) p_{1}(V)\right]}-1}{p_{1}(T M)-(a+2 b) p_{1}(V)} \widehat{A}(T M) \operatorname{ch}\left((\triangle(V))^{b}\right) \operatorname{ch}\left(-a \widetilde{V_{\mathbf{C}}}-24 k\right)\right\}^{(4 k-4)} .
\end{gathered}
$$

Our main results in this section include the following theorem.

\section{Theorem 3.1}

$$
\begin{gathered}
\left\{\widehat{A}\left(T M, \nabla^{T M}\right) \operatorname{ch}\left((\triangle(V))^{a}\right)\right\}^{(4 k)} \\
-\sum_{r=0}^{\left[\frac{k}{2}\right]} 2^{(a-b) l+k-6 r}\left\{\widehat{A}\left(T M, \nabla^{T M}\right) \operatorname{ch}\left((\triangle(V))^{b}\right) \operatorname{ch}\left(b_{r}\right)\right\}^{(4 k)} \\
=\left[p_{1}(T M)-(a+2 b) p_{1}(V)\right] \mathcal{B}\left(\nabla^{T M}, \nabla^{V}, a, b\right),
\end{gathered}
$$

where

$$
\begin{gathered}
\mathcal{B}\left(\nabla^{T M}, \nabla^{V}, a, b\right)=\sum_{r=0}^{\left[\frac{k}{2}\right]} 2^{(a-b) l+k-6 r} \beta_{r} \\
-\left\{\frac{e^{\frac{1}{24}\left[p_{1}(T M)-(a+2 b) p_{1}(V)\right]}-1}{p_{1}(T M)-(a+2 b) p_{1}(V)} \widehat{A}(T M) \operatorname{ch}\left((\triangle(V))^{a}\right)\right\}^{(4 k-4)}
\end{gathered}
$$


Proof. Let $\left\{ \pm 2 \pi \sqrt{-1} x_{j} \mid 1 \leq j \leq 2 k\right\}$ be the Chern roots of $T_{C} M$. Set

$$
\begin{gathered}
Q_{1}(\tau)=\left\{e^{\frac{1}{24} E_{2}(\tau)\left[p_{1}(T M)-(a+2 b) p_{1}(V)\right]} \widehat{A}\left(T M, \nabla^{T M}\right) \operatorname{ch}\left((\triangle(V))^{a}\right) \operatorname{ch}\left(\Theta_{1}\left(T_{C} M, V_{C}, a, b\right)\right)\right\}^{(4 k)} \\
Q_{2}(\tau)=\left\{\widehat{A}\left(T M, \nabla^{T M}\right) \operatorname{ch}\left((\triangle(V))^{b}\right) \operatorname{ch}\left(\Theta_{2}\left(T_{C} M, V_{C}, a, b\right)\right)\right\}^{(4 k)}, \\
\overline{Q_{2}(\tau)}=\left\{\frac{e^{\frac{1}{24} E_{2}(\tau)\left[p_{1}(T M)-(a+2 b) p_{1}(V)\right]}-1}{p_{1}(T M)-(a+2 b) p_{1}(V)}\right. \\
\left.\widehat{A}\left(T M, \nabla^{T M}\right) \operatorname{ch}\left((\triangle(V))^{b}\right) \operatorname{ch}\left(\Theta_{2}\left(T_{C} M, V_{C}, a, b\right)\right)\right\}^{(4 k-4)}
\end{gathered}
$$

Direct computations show that

$$
\begin{aligned}
Q_{1}(\tau)= & 2^{a l}\left\{e^{\frac{1}{24} E_{2}(\tau)\left[p_{1}(T M)-(a+2 b) p_{1}(V)\right]}\left(\prod_{j=1}^{2 k} \frac{x_{j} \theta^{\prime}(0, \tau)}{\theta\left(x_{j}, \tau\right)}\right)\right. \\
& \left.\cdot\left(\prod_{\nu=1}^{l} \frac{\theta_{1}^{a}\left(y_{\nu}, \tau\right)}{\theta_{1}^{a}(0, \tau)} \frac{\theta_{2}^{b}\left(y_{\nu}, \tau\right)}{\theta_{2}^{b}(0, \tau)} \frac{\theta_{3}^{b}\left(y_{\nu}, \tau\right)}{\theta_{3}^{b}(0, \tau)}\right)\right\}^{(4 k)}
\end{aligned}
$$

Similarly, we have

$$
\begin{gathered}
Q_{2}(\tau)+\left[p_{1}(T M)-(a+2 b) p_{1}(V)\right] \overline{Q_{2}(\tau)} \\
=2^{b l}\left\{e^{\frac{1}{24} E_{2}(\tau)\left[p_{1}(T M)-(a+2 b) p_{1}(V)\right]}\left(\prod_{j=1}^{2 k} \frac{x_{j} \theta^{\prime}(0, \tau)}{\theta\left(x_{j}, \tau\right)}\right)\right. \\
\left.\cdot\left(\prod_{\nu=1}^{l} \frac{\theta_{2}^{a}\left(y_{\nu}, \tau\right)}{\theta_{2}^{a}(0, \tau)} \frac{\theta_{1}^{b}\left(y_{\nu}, \tau\right)}{\theta_{1}^{b}(0, \tau)} \frac{\theta_{3}^{b}\left(y_{\nu}, \tau\right)}{\theta_{3}^{b}(0, \tau)}\right)\right\}^{(4 k)} .
\end{gathered}
$$

By (2.12)-(2.16) and (2.19)-(2.20), then $Q_{1}(\tau)$ is a modular form of weight $2 k$ over $\Gamma_{0}(2)$, while $Q_{2}(\tau)+\left[p_{1}(T M)-(a+2 b) p_{1}(V)\right] \overline{Q_{2}(\tau)}$ is a modular form of weight $2 k$ over $\Gamma^{0}(2)$. Moreover, the following identity holds,

$$
Q_{1}\left(-\frac{1}{\tau}\right)=2^{(a-b) l} \tau^{2 k}\left(Q_{2}(\tau)+\left[p_{1}(T M)-(a+2 b) p_{1}(V)\right] \overline{Q_{2}(\tau)}\right) .
$$

Observe that at any point $x \in M$, up to the volume form determined by the metric on $T_{x} M$, both $Q_{i}(\tau)$, and $Q_{2}(\tau)+\left[p_{1}(T M)-(a+2 b) p_{1}(V)\right] \overline{Q_{2}(\tau)}$ can be view as a power series of $q^{\frac{1}{2}}$ with real Fourier coefficients. By Lemma 2.2, we have

$Q_{2}(\tau)+\left[p_{1}(T M)-(a+2 b) p_{1}(V)\right] \overline{Q_{2}(\tau)}=h_{0}\left(8 \delta_{2}\right)^{k}+h_{1}\left(8 \delta_{2}\right)^{k-2} \varepsilon_{2}+\cdots+h_{\left[\frac{k}{2}\right]}\left(8 \delta_{2}\right)^{k-2\left[\frac{k}{2}\right]} \varepsilon_{2}^{\left[\frac{k}{2}\right]}$,

where each $h_{r}, 0 \leq r \leq\left[\frac{k}{2}\right]$, is a real multiple of the volume form at $x$. By the definitions of $b_{r}$ and $\beta_{r}$, we have

$$
h_{r}=\left\{\widehat{A}\left(T M, \nabla^{T M}\right) \operatorname{ch}\left((\triangle(V))^{b}\right) \operatorname{ch}\left(b_{r}\right)\right\}^{(4 k)}+\left[p_{1}(T M)-(a+2 b) p_{1}(V)\right] \beta_{r} .
$$


By (2.21) (3.15) and (3.16), we get

$$
Q_{1}(\tau)=2^{(a-b) l}\left[h_{0}\left(8 \delta_{1}\right)^{k}+h_{1}\left(8 \delta_{1}\right)^{k-2} \varepsilon_{1}+\cdots+h_{\left[\frac{k}{2}\right]}\left(8 \delta_{1}\right)^{k-2\left[\frac{k}{2}\right]} \varepsilon_{1}^{\left[\frac{k}{2}\right]}\right] .
$$

By comparing the constant term in (3.18), we get

$$
\begin{gathered}
\left\{e^{\frac{1}{24} E_{2}(\tau)\left[p_{1}(T M)-(a+2 b) p_{1}(V)\right]} \widehat{A}\left(T M, \nabla^{T M}\right) \operatorname{ch}\left((\triangle(V))^{a}\right)\right\}^{(4 k)} \\
=\sum_{r=0}^{\left[\frac{k}{2}\right]} 2^{(a-b) l+k-6 r}\left(\left\{\widehat{A}\left(T M, \nabla^{T M}\right) \operatorname{ch}\left((\triangle(V))^{b}\right) \operatorname{ch}\left(b_{r}\right)\right\}^{(4 k)}+\left[p_{1}(T M)-(a+2 b) p_{1}(V)\right] \beta_{r}\right),
\end{gathered}
$$

So we get Theorem 3.1.

Remark. By Theorem 3.1, if $M$ is a $4 k$ dimensional spin manifolds and $V$ is a $2 l$ dimensional spin vector bundle over $M$, then we have

$$
\int_{M}\left[p_{1}(T M)-(a+2 b) p_{1}(V)\right] \mathcal{B}\left(\nabla^{T M}, \nabla^{V}, a, b\right),
$$

is an integer. When $a=1, b=0$, we get the Han-Liu-Zhang cancellation formula.

By the direct computations, we have

Corollary 3.2 When $\operatorname{dim} M=4$, the following identity holds

$$
\begin{gathered}
\left\{\widehat{A}\left(T M, \nabla^{T M}\right) \operatorname{ch}\left((\triangle(V))^{a}\right)\right\}^{(4)}+2^{(a-b) l+1}\left\{\widehat{A}\left(T M, \nabla^{T M}\right) \operatorname{ch}\left((\triangle(V))^{b}\right)\right\}^{(4)} \\
=-2^{a l-3}\left[p_{1}(T M)-(a+2 b) p_{1}(V)\right] .
\end{gathered}
$$

Corollary 3.3 When $\operatorname{dim} M=8$, the following identity holds

$$
\begin{gathered}
\left\{\widehat{A}\left(T M, \nabla^{T M}\right) \operatorname{ch}\left((\triangle(V))^{a}\right)\right\}^{(8)} \\
-\left\{-2^{(a-b) l-4} a \widehat{A}\left(T M, \nabla^{T M}\right) \operatorname{ch}\left((\triangle(V))^{b}\right) \operatorname{ch}\left(\widetilde{V_{\mathbf{C}}}\right)+2^{(a-b) l} \widehat{A}\left(T M, \nabla^{T M}\right) \operatorname{ch}\left((\triangle(V))^{b}\right)\right\}^{(8)} \\
=\left[p_{1}(T M)-(a+2 b) p_{1}(V)\right]\left\{\frac{e^{\frac{1}{24}\left[p_{1}(T M)-(a+2 b) p_{1}(V)\right]}-1}{p_{1}(T M)-(a+2 b) p_{1}(V)}\right. \\
{\left[2^{(a-b) l} \widehat{A}\left(T M, \nabla^{T M}\right) \operatorname{ch}\left((\triangle(V))^{b}\right)-a \widehat{A}\left(T M, \nabla^{T M}\right) \operatorname{ch}\left((\triangle(V))^{b}\right) \operatorname{ch}\left(\widetilde{V_{\mathbf{C}}}\right)\right.} \\
\left.\left.-\widehat{A}\left(T M, \nabla^{T M}\right) \operatorname{ch}\left((\triangle(V))^{a}\right)\right]\right\}^{(8)}
\end{gathered}
$$

Let $\xi$ be a rank two real oriented Euclidean vector bundle over $M$ carrying with an Euclidean connection $\nabla^{\xi}$. Set

$$
\Theta_{1}\left(T_{C} M, V_{C}, \xi_{C}, a, b\right)=\bigotimes_{n=1}^{\infty} S_{q^{n}}\left(\widetilde{T_{C} M}\right) \otimes \bigotimes_{m=1}^{\infty} \wedge_{q^{m}}\left(a \widetilde{V_{C}}-2 \widetilde{\xi_{C}}\right)
$$




$$
\begin{gathered}
\otimes \bigotimes_{r=1}^{\infty} \wedge_{q^{r-\frac{1}{2}}}\left(b \widetilde{V_{C}}+\widetilde{\xi_{C}}\right) \otimes \bigotimes_{s=1}^{\infty} \wedge_{-q^{s-\frac{1}{2}}}\left(b \widetilde{V_{C}}+\widetilde{\xi_{C}}\right), \\
\Theta_{2}\left(T_{C} M, V_{C}, \xi_{C}, a, b\right)=\bigotimes_{n=1}^{\infty} S_{q^{n}}\left(\widetilde{T_{C} M}\right) \otimes \bigotimes_{m=1}^{\infty} \wedge_{q^{m}}\left(b \widetilde{V_{C}}+\widetilde{\xi_{C}}\right) \\
\otimes \bigotimes_{r=1}^{\infty} \wedge_{q^{r-\frac{1}{2}}}\left(b \widetilde{V_{C}}+\widetilde{\xi_{C}}\right) \otimes \bigotimes_{s=1}^{\infty} \wedge_{-q^{s-\frac{1}{2}}}\left(a \widetilde{V_{C}}-2 \widetilde{\xi_{C}}\right),
\end{gathered}
$$

Clearly, $\Theta_{1}\left(T_{C} M, V_{C}, \xi_{C}, a, b\right)$ and $\Theta_{2}\left(T_{C} M, V_{C}, \xi_{C}, a, b\right)$ admit formal Fourier expansion in $q^{\frac{1}{2}}$ as

$$
\begin{aligned}
& \Theta_{1}\left(T_{C} M, V_{C}, \xi_{C}, a, b\right)=A_{0}\left(T_{C} M, V_{C}, \xi_{C}, a, b\right)+A_{1}\left(T_{C} M, V_{C}, \xi_{C}, a, b\right) q^{\frac{1}{2}}+\cdots, \\
& \Theta_{2}\left(T_{C} M, V_{C}, \xi_{C}, a, b\right)=B_{0}\left(T_{C} M, V_{C}, \xi_{C}, a, b\right)+B_{1}\left(T_{C} M, V_{C}, \xi_{C}, a, b\right) q^{\frac{1}{2}}+\cdots,
\end{aligned}
$$

Let $c=2 \pi \sqrt{-1} u$ be the Euler form of $\xi$. Set

$$
\begin{gathered}
\widetilde{Q}_{1}(\tau)=\left\{\frac{e^{\frac{1}{24} E_{2}(\tau)\left[p_{1}(T M)-(a+2 b) p_{1}(V)\right]} \widehat{A}\left(T M, \nabla^{T M}\right) \operatorname{ch}\left((\triangle(V))^{a}\right)}{\cosh ^{2}\left(\frac{c}{2}\right)} \operatorname{ch}\left(\Theta_{1}\left(T_{C} M, V_{C}, \xi_{C}, a, b\right)\right)\right\}^{(4 k)} \\
\widetilde{Q}_{2}(\tau)=\left\{\widehat{A}\left(T M, \nabla^{T M}\right) \cosh \left(\frac{c}{2}\right) \operatorname{ch}\left((\triangle(V))^{b}\right) \operatorname{ch}\left(\Theta_{2}\left(T_{C} M, V_{C}, \xi_{C}, a, b\right)\right)\right\}^{(4 k)}, \\
\widetilde{Q}_{3}(\tau)=\left\{\frac{e^{\frac{1}{24} E_{2}(\tau)\left[p_{1}(T M)-(a+2 b) p_{1}(V)\right]}-1}{p_{1}(T M)-(a+2 b) p_{1}(V)}\right. \\
\left.\cdot \widehat{A}\left(T M, \nabla^{T M}\right) \cosh \left(\frac{c}{2}\right) \operatorname{ch}\left((\triangle(V))^{b}\right) \operatorname{ch}\left(\Theta_{2}\left(T_{C} M, V_{C}, \xi_{C}, a, b\right)\right)\right\}^{(4 k)}
\end{gathered}
$$

Define virtual complex vector bundle $\widetilde{b}_{r}\left(T_{\mathbf{C}} M, V_{\mathbf{C}}, a, b\right)$ on $M, 0 \leq r \leq\left[\frac{k}{2}\right]$, via the equality

$$
\Theta_{2}\left(T_{C} M, V_{C}, \xi_{C}, a, b\right) \equiv \sum_{r=0}^{\left[\frac{k}{2}\right]} \widetilde{b}_{r}\left(8 \delta_{2}\right)^{k-2 r} \varepsilon_{2}^{r} \quad \bmod q^{\frac{\left[\frac{k}{2}\right]+1}{2}} \dot{K}(M)\left[\left[q^{\frac{1}{2}}\right]\right] .
$$

Define degree $4 k-4$ differential forms $\widetilde{\beta}_{r}\left(T_{\mathbf{C}} M, V_{\mathbf{C}}, \xi_{C}, a, b\right)$ on $M, 0 \leq r \leq\left[\frac{k}{2}\right]$, via the equality

$$
\begin{gathered}
\left\{\frac{e^{\frac{1}{24} E_{2}(\tau)\left[p_{1}(T M)-(a+2 b) p_{1}(V)\right]}-1}{p_{1}(T M)-(a+2 b) p_{1}(V)} \widehat{A}(T M) \operatorname{ch}\left((\triangle(V))^{b}\right) \cosh \left(\frac{c}{2}\right) \operatorname{ch}\left(\Theta_{2}\left(T_{C} M, V_{C}, \xi_{C}, a, b\right)\right)\right\}^{(4 k-4)} \\
\equiv \sum_{r=0}^{\left[\frac{k}{2}\right]} \beta_{r}\left(8 \delta_{2}\right)^{k-2 r} \varepsilon_{2}^{r} \quad \bmod q^{\frac{\left[\frac{k}{2}\right]+1}{2}} \dot{\Omega}^{(4 k-4)}(M)\left[\left[q^{\frac{1}{2}}\right]\right]
\end{gathered}
$$

Then similar to Theorem 3.1, we get 
Theorem 3.4

$$
\begin{gathered}
\left\{\frac{\widehat{A}\left(T M, \nabla^{T M}\right) \operatorname{ch}\left((\triangle(V))^{a}\right)}{\cosh ^{2}\left(\frac{c}{2}\right)}\right\}^{(4 k)} \\
-\sum_{r=0}^{\left[\frac{k}{2}\right]} 2^{(a-b) l+k-6 r}\left\{\widehat{A}\left(T M, \nabla^{T M}\right) \cosh \left(\frac{c}{2}\right) \operatorname{ch}\left((\triangle(V))^{b}\right) \operatorname{ch}\left(\widetilde{b}_{r}\right)\right\}^{(4 k)} \\
=\left[p_{1}(T M)-(a+2 b) p_{1}(V)\right] \widetilde{\mathcal{B}}\left(\nabla^{T M}, \nabla^{V}, a, b\right),
\end{gathered}
$$

where

$$
\begin{gathered}
\widetilde{\mathcal{B}}\left(\nabla^{T M}, \nabla^{V}, \nabla^{\xi}, a, b\right)=\sum_{r=0}^{\left[\frac{k}{2}\right]} 2^{(a-b) l+k-6 r \widetilde{\beta_{r}}} \\
-\left\{\frac{e^{\frac{1}{24}\left[p_{1}(T M)-(a+2 b) p_{1}(V)\right]}-1}{p_{1}(T M)-(a+2 b) p_{1}(V)} \frac{\widehat{A}\left(T M, \nabla^{T M}\right) \operatorname{ch}\left((\triangle(V))^{a}\right)}{\cosh ^{2}\left(\frac{c}{2}\right)}\right\}^{(4 k-4)} .
\end{gathered}
$$

\section{The anomaly cancellation formulas involving two com- plex line bundles}

Let $\xi, \xi^{\prime}$ be two rank two real Euclidean vector bundle with Euclidean connections $\nabla^{\xi}, \nabla^{\xi^{\prime}}$. Set

$$
\begin{gathered}
\Theta_{1}\left(T_{C} M, V_{C}, \xi_{C}, \xi_{C}^{\prime}\right)=\bigotimes_{n=1}^{\infty} S_{q^{n}}\left(\widetilde{T_{C} M}\right) \otimes \bigotimes_{m=1}^{\infty} \wedge_{q^{m}}\left(\widetilde{V_{C}}-2 \widetilde{\xi_{C}}\right) \\
\otimes \bigotimes_{r=1}^{\infty} \wedge_{q^{r-\frac{1}{2}}}\left(\widetilde{\xi_{C}^{\prime}}\right) \otimes \bigotimes_{s=1}^{\infty} \wedge_{-q^{s-\frac{1}{2}}}\left(\widetilde{\xi_{C}^{\prime}}\right), \\
\Theta_{2}\left(T_{C} M, V_{C}, \xi_{C}, \xi_{C}^{\prime}\right)=\bigotimes_{n=1}^{\infty} S_{q^{n}}\left(\widetilde{T_{C} M}\right) \otimes \bigotimes_{m=1}^{\infty} \wedge_{q^{m}}\left(\widetilde{\xi_{C}^{\prime}}\right) \\
\otimes \bigotimes_{r=1}^{\infty} \wedge_{q^{r-\frac{1}{2}}}\left(\widetilde{\xi_{C}^{\prime}}\right) \otimes \bigotimes_{s=1}^{\infty} \wedge_{-q^{s-\frac{1}{2}}}\left(\widetilde{V_{C}}-2 \widetilde{\xi_{C}}\right),
\end{gathered}
$$

Set

$$
\begin{gathered}
P_{1}(\tau)=\left\{\frac{e^{\frac{1}{12} E_{2}(\tau)\left[p_{1}(\xi)-p_{1}\left(\xi^{\prime}\right)\right]} \widehat{A}\left(T M, \nabla^{T M}\right) \operatorname{ch}(\triangle(V))}{\cosh ^{2}\left(\frac{c}{2}\right)} \operatorname{ch}\left(\Theta_{1}\left(T_{C} M, V_{C}, \xi_{C}, \xi_{C}^{\prime}\right)\right)\right\}_{(4.2)}^{(4 k)}, \\
P_{2}(\tau)=\left\{\widehat{A}\left(T M, \nabla^{T M}\right) \cosh \left(\frac{c^{\prime}}{2}\right) \operatorname{ch}\left(\Theta_{2}\left(T_{C} M, V_{C}, \xi_{C}, \xi_{C}^{\prime}\right)\right)\right\}^{(4 k)}, \\
P_{3}(\tau)=\left\{\frac{e^{\frac{1}{12} E_{2}(\tau)\left[p_{1}(\xi)-p_{1}\left(\xi^{\prime}\right)\right]}-1}{p_{1}(\xi)-p_{1}\left(\xi^{\prime}\right)}\right.
\end{gathered}
$$




$$
\left.\cdot \widehat{A}\left(T M, \nabla^{T M}\right) \cosh \left(\frac{c^{\prime}}{2}\right) \operatorname{ch}\left(\Theta_{2}\left(T_{C} M, V_{C}, \xi_{C}, \xi_{C}^{\prime}\right)\right)\right\}^{(4 k)},
$$

Define virtual complex vector bundle $\bar{b}_{r}\left(T_{\mathbf{C}} M, V_{\mathbf{C}}, \xi, \xi^{\prime}\right)$ on $M, 0 \leq r \leq\left[\frac{k}{2}\right]$, via the equality

$$
\Theta_{2}\left(T_{C} M, V_{C}, \xi, \xi^{\prime}\right) \equiv \sum_{r=0}^{\left[\frac{k}{2}\right]} \bar{b}_{r}\left(8 \delta_{2}\right)^{k-2 r} \varepsilon_{2}^{r} \quad \bmod q^{\frac{\left[\frac{k}{2}\right]+1}{2}} \dot{K}(M)\left[\left[q^{\frac{1}{2}}\right]\right] .
$$

Then

$$
\bar{b}_{0}=(-1)^{k} \mathbf{C}, \quad \bar{b}_{1}=-24(-1)^{k} k+2 \widetilde{\xi_{C}}+\widetilde{\xi_{C}}-\widetilde{V_{\mathbf{C}}} .
$$

Define degree $4 k-4$ differential forms $\bar{\beta}_{r}\left(T_{\mathbf{C}} M, V_{\mathbf{C}}, \xi, \xi^{\prime}\right)$ on $M, 0 \leq r \leq\left[\frac{k}{2}\right]$, via the equality

$$
\begin{gathered}
\left\{\frac{e^{\frac{1}{12} E_{2}(\tau)\left[p_{1}(\xi)-p_{1}\left(\xi^{\prime}\right)\right]}-1}{p_{1}(\xi)-p_{1}\left(\xi^{\prime}\right)} \widehat{A}(T M) \cosh \left(\frac{c^{\prime}}{2}\right) \operatorname{ch}\left(\Theta_{2}\left(T_{C} M, V_{C}, \xi, \xi^{\prime}\right)\right)\right\}^{(4 k-4)} \\
\equiv \sum_{r=0}^{\left[\frac{k}{2}\right]} \bar{\beta}_{r}\left(8 \delta_{2}\right)^{k-2 r} \varepsilon_{2}^{r} \quad \bmod q^{\frac{\left[\frac{k}{2}\right]+1}{2}} \dot{\Omega}^{(4 k-4)}(M)\left[\left[q^{\frac{1}{2}}\right]\right]
\end{gathered}
$$

It is easy to calculate that

$$
\begin{gathered}
\bar{\beta}_{0}=(-1)^{k}\left\{\frac{e^{\frac{1}{12}\left[p_{1}(\xi)-p_{1}\left(\xi^{\prime}\right)\right]}-1}{p_{1}(\xi)-p_{1}\left(\xi^{\prime}\right)} \widehat{A}(T M) \cosh \left(\frac{c^{\prime}}{2}\right)\right\}^{(4 k-4)}, \\
\bar{\beta}_{1}=(-1)^{k}\left\{\frac{e^{\frac{1}{12}\left[p_{1}(\xi)-p_{1}\left(\xi^{\prime}\right)\right]}-1}{p_{1}(\xi)-p_{1}\left(\xi^{\prime}\right)} \widehat{A}(T M) \cosh \left(\frac{c^{\prime}}{2}\right) \operatorname{ch}\left(-24 k+2 \widetilde{\xi_{C}}+\widetilde{\xi}^{\prime}-\widetilde{V_{\mathbf{C}}}\right)\right\}^{(4 k-4)},
\end{gathered}
$$

We have

$$
\begin{gathered}
P_{1}(\tau)=2^{l}\left\{e^{\frac{1}{12} E_{2}(\tau)\left[p_{1}(\xi)-p_{1}\left(\xi^{\prime}\right)\right]}\left(\prod_{j=1}^{2 k} \frac{x_{j} \theta^{\prime}(0, \tau)}{\theta\left(x_{j}, \tau\right)}\right)\left(\prod_{\nu=1}^{l} \frac{\theta_{1}\left(y_{\nu}, \tau\right)}{\theta_{1}(0, \tau)}\right)\right. \\
\left.\cdot\left(\frac{\theta_{1}^{2}(0, \tau)}{\theta_{1}^{2}(u, \tau)} \frac{\theta_{3}\left(u^{\prime}, \tau\right)}{\theta_{3}(0, \tau)} \frac{\theta_{2}\left(u^{\prime}, \tau\right)}{\theta_{2}(0, \tau)}\right)\right\}^{(4 k)} .
\end{gathered}
$$

Similarly,

$$
\begin{gathered}
P_{2}(\tau)+\left[p_{1}(\xi)-p_{1}\left(\xi^{\prime}\right)\right] P_{3}(\tau)=\left\{e^{\frac{1}{12} E_{2}(\tau)\left[p_{1}(\xi)-p_{1}\left(\xi^{\prime}\right)\right]}\left(\prod_{j=1}^{2 k} \frac{x_{j} \theta^{\prime}(0, \tau)}{\theta\left(x_{j}, \tau\right)}\right)\right. \\
\left.\cdot\left(\prod_{\nu=1}^{l} \frac{\theta_{2}\left(y_{\nu}, \tau\right)}{\theta_{2}(0, \tau)}\right)\left(\frac{\theta_{2}^{2}(0, \tau)}{\theta_{2}^{2}(u, \tau)} \frac{\theta_{3}\left(u^{\prime}, \tau\right)}{\theta_{3}(0, \tau)} \frac{\theta_{1}\left(u^{\prime}, \tau\right)}{\theta_{1}(0, \tau)}\right)\right\}^{(4 k)} \cdot
\end{gathered}
$$


We assume that $p_{1}(T M)=p_{1}(V)$, then we have $P_{1}(\tau)$ is a modular form of weight $2 k$ over $\Gamma_{0}(2)$, while $P_{2}(\tau)+\left[p_{1}(\xi)-p_{1}\left(\xi^{\prime}\right)\right] P_{3}(\tau)$ is a modular form of weight $2 k$ over $\Gamma^{0}(2)$. Moreover, the following identity holds,

$$
P_{1}\left(-\frac{1}{\tau}\right)=2^{l} \tau^{2 k}\left(P_{2}(\tau)+\left[p_{1}(\xi)-p_{1}\left(\xi^{\prime}\right)\right] P_{3}(\tau)\right) .
$$

So similar to the discussions in Section 3, we get

Theorem 4.1 If $p_{1}(T M)=p_{1}(V)$, then

$$
\begin{gathered}
\left\{\frac{\widehat{A}\left(T M, \nabla^{T M}\right) \operatorname{ch}(\triangle(V))}{\cosh ^{2}\left(\frac{c}{2}\right)}\right\}^{(4 k)} \\
-\sum_{r=0}^{\left[\frac{k}{2}\right]} 2^{l+k-6 r}\left\{\widehat{A}\left(T M, \nabla^{T M}\right) \cosh \left(\frac{c^{\prime}}{2}\right) \operatorname{ch}\left(\bar{b}_{r}\right)\right\}^{(4 k)} \\
=\left[p_{1}(\xi)-p_{1}\left(\xi^{\prime}\right)\right] \overline{\mathcal{B}}\left(\nabla^{T M}, \nabla^{V}, \nabla^{\xi}, \nabla^{\xi^{\prime}}\right),
\end{gathered}
$$

where

$$
\begin{gathered}
\widetilde{\mathcal{B}}\left(\nabla^{T M}, \nabla^{V}, \nabla^{\xi}, \nabla^{\xi^{\prime}}\right)=\sum_{r=0}^{\left[\frac{k}{2}\right]} 2^{l+k-6 r \overline{\beta_{r}}} \\
-\left\{\frac{e^{\frac{1}{12}\left[p_{1}(\xi)-p_{1}\left(\xi^{\prime}\right)\right]}-1}{p_{1}(\xi)-p_{1}\left(\xi^{\prime}\right)} \frac{\widehat{A}\left(T M, \nabla^{T M}\right) \operatorname{ch}(\triangle(V))}{\cosh ^{2}\left(\frac{c}{2}\right)}\right\}^{(4 k-4)} .
\end{gathered}
$$

Corollary 4.2 When $\operatorname{dim} M=4$, the following identity holds

$$
\begin{gathered}
\left\{\frac{\widehat{A}\left(T M, \nabla^{T M}\right) \operatorname{ch}(\triangle(V))}{\cosh ^{2}\left(\frac{c}{2}\right)}\right\}^{(4)}+2^{l+1}\left\{\widehat{A}\left(T M, \nabla^{T M}\right) \cosh \left(\frac{c^{\prime}}{2}\right)\right\}^{(4)} \\
=-2^{l-2}\left[p_{1}(\xi)-p_{1}\left(\xi^{\prime}\right)\right] .
\end{gathered}
$$

Corollary 4.3 When $\operatorname{dim} M=8$, the following identity holds

$$
\begin{gathered}
\left\{\frac{\widehat{A}\left(T M, \nabla^{T M}\right) \operatorname{ch}(\triangle(V))}{\cosh ^{2}\left(\frac{c}{2}\right)}\right\}^{(8)}-2^{l}\left\{\widehat{A}\left(T M, \nabla^{T M}\right) \cosh \left(\frac{c^{\prime}}{2}\right)\right\}^{(8)} \\
-2^{l-4}\left\{\widehat{A}\left(T M, \nabla^{T M}\right) \cosh \left(\frac{c^{\prime}}{2}\right) \operatorname{ch}\left(2 \widetilde{\xi_{C}}+\widetilde{\xi_{C}^{\prime}}-\widetilde{V_{C}}\right)\right\}^{(8)} \\
=\left[p_{1}(\xi)-p_{1}\left(\xi^{\prime}\right)\right]\left\{\frac{e^{\frac{1}{12}\left[p_{1}(\xi)-p_{1}\left(\xi^{\prime}\right)\right]}-1}{p_{1}(\xi)-p_{1}\left(x i^{\prime}\right)}\right.
\end{gathered}
$$




$$
\begin{gathered}
\cdot\left[2^{l} \widehat{A}\left(T M, \nabla^{T M}\right)\right. \\
\cosh \left(\frac{c^{\prime}}{2}\right)-\widehat{A}\left(T M, \nabla^{T M}\right) \cosh \left(\frac{c^{\prime}}{2}\right) \operatorname{ch}\left(2 \widetilde{\xi_{C}}+\widetilde{\xi_{C}}-\widetilde{V_{\mathbf{C}}}\right) \\
\left.\left.-\frac{\widehat{A}\left(T M, \nabla^{T M}\right) \operatorname{ch}(\triangle(V))}{\cosh ^{2}\left(\frac{c}{2}\right)}\right]\right\}^{(8)}
\end{gathered}
$$

\section{The odd dimensional case}

In this section, Let $M$ be a (4k-1)-dimensional manifold. In the definition (3.22), we set $a=1, b=0$ and $\Phi_{1}\left(\nabla^{T M}, \nabla^{V}, \nabla^{\xi}, \tau\right)=\widetilde{Q_{1}}(\tau)$, and $\Phi_{2}\left(\nabla^{T M}, \nabla^{V}, \nabla^{\xi}, \tau\right)=$ $\widetilde{Q_{2}}(\tau)+\left[p_{1}(T M)-p_{1}(V)\right] \widetilde{Q_{3}(\tau)}$. Applying the Chern-Weil theory, we can express $\Phi_{1}, \Phi_{2}$ as follows:

$$
\begin{gathered}
\Phi_{1}\left(\nabla^{T M}, \nabla^{V}, \nabla^{\xi}, \tau\right)=2^{l} e^{\frac{1}{24} E_{2}(\tau)\left[p_{1}(T M)-p_{1}(V)\right]} \operatorname{det}^{\frac{1}{2}}\left(\frac{R^{T M}}{4 \pi^{2}} \frac{\theta^{\prime}(0, \tau)}{\theta\left(\frac{R^{T M}}{4 \pi^{2}}, \tau\right)}\right) \\
\cdot \operatorname{det}^{\frac{1}{2}}\left(\frac{\theta_{1}\left(\frac{R^{V}}{4 \pi^{2}}, \tau\right)}{\theta_{1}(0, \tau)}\right) \operatorname{det}^{\frac{1}{2}}\left(\frac{\theta_{1}^{2}(0, \tau)}{\theta_{1}^{2}\left(\frac{R^{\xi}}{4 \pi^{2}}, \tau\right)} \frac{\theta_{3}\left(\frac{R^{\xi}}{4 \pi^{2}}, \tau\right)}{\theta_{3}(0, \tau)} \frac{\theta_{2}\left(\frac{R^{\xi}}{4 \pi^{2}}, \tau\right)}{\theta_{2}(0, \tau)}\right) ; \\
\Phi_{2}\left(\nabla^{T M}, \nabla^{V}, \nabla^{\xi}, \tau\right)=e^{\frac{1}{24} E_{2}(\tau)\left[p_{1}(T M)-p_{1}(V)\right]} \operatorname{det}^{\frac{1}{2}}\left(\frac{R^{T M}}{4 \pi^{2}} \frac{\theta^{\prime}(0, \tau)}{\theta\left(\frac{R^{T M}}{4 \pi^{2}}, \tau\right)}\right) \\
\cdot \operatorname{det}^{\frac{1}{2}}\left(\frac{\theta_{2}\left(\frac{R^{V}}{4 \pi^{2}}, \tau\right)}{\theta_{2}(0, \tau)}\right) \operatorname{det} \frac{1}{2}\left(\frac{\theta_{2}^{2}(0, \tau)}{\theta_{2}^{2}\left(\frac{R^{\xi}}{4 \pi^{2}}, \tau\right)} \frac{\theta_{3}\left(\frac{R^{\xi}}{4 \pi^{2}}, \tau\right)}{\theta_{3}(0, \tau)} \frac{\theta_{1}\left(\frac{R^{\xi}}{4 \pi^{2}}, \tau\right)}{\theta_{1}(0, \tau)}\right)
\end{gathered}
$$

Next we consider the transgression of $\Phi_{1}\left(\nabla^{T M}, \nabla^{V}, \nabla^{\xi}, \tau\right), \Phi_{1}\left(\nabla^{T M}, \nabla^{V}, \nabla^{\xi}, \tau\right)$. about $\nabla^{\xi}$. Let $\nabla_{1}^{\xi}, \nabla_{0}^{\xi}$ be two Euclidean connections on $\xi$ and $B=\nabla_{1}^{\xi}-\nabla_{0}^{\xi}, \nabla_{t}^{\xi}=$ $t \nabla_{1}^{\xi}+(1-t) \nabla_{0}^{\xi}$. We have

$$
\begin{aligned}
& \Phi_{1}\left(\nabla^{T M}, \nabla^{V}, \nabla_{1}^{\xi}, \tau\right)-\Phi_{1}\left(\nabla^{T M}, \nabla^{V}, \nabla_{0}^{\xi}, \tau\right) \\
& =\frac{1}{8 \pi^{2}} d \int_{0}^{1} \Phi_{1}\left(\nabla^{T M}, \nabla^{V}, \nabla_{t}^{\xi}, \tau\right) \operatorname{tr}\left[B\left(\frac{\theta_{2}^{\prime}\left(\frac{R_{t}^{\xi}}{4 \pi^{2}}, \tau\right)}{\theta_{2}\left(\frac{R_{t}^{\xi}}{4 \pi^{2}}, \tau\right)}+\frac{\theta_{3}^{\prime}\left(\frac{R_{t}^{\xi}}{4 \pi^{2}}, \tau\right)}{\theta_{3}\left(\frac{R_{t}^{\xi}}{4 \pi^{2}}, \tau\right)}-2 \frac{\theta_{1}^{\prime}\left(\frac{R_{t}^{\xi}}{4 \pi^{2}}, \tau\right)}{\theta_{1}\left(\frac{R_{t}^{\xi}}{4 \pi^{2}}, \tau\right)}\right)\right] d t .
\end{aligned}
$$

We define

$$
\begin{aligned}
& C S \Phi_{1}\left(\nabla^{T M}, \nabla^{V}, \nabla_{0}^{\xi}, \nabla_{1}^{\xi}, \tau\right) \\
& :=\frac{\sqrt{2}}{8 \pi^{2}} \int_{0}^{1} \Phi_{1}\left(\nabla^{T M}, \nabla^{V}, \nabla_{t}^{\xi}, \tau\right) \operatorname{tr}\left[B\left(\frac{\theta_{2}^{\prime}\left(\frac{R_{t}^{\xi}}{4 \pi^{2}}, \tau\right)}{\theta_{2}\left(\frac{R_{t}^{\xi}}{4 \pi^{2}}, \tau\right)}+\frac{\theta_{3}^{\prime}\left(\frac{R_{t}^{\xi}}{4 \pi^{2}}, \tau\right)}{\theta_{3}\left(\frac{R_{t}^{\xi}}{4 \pi^{2}}, \tau\right)}-2 \frac{\theta_{1}^{\prime}\left(\frac{R_{t}^{\xi}}{4 \pi^{2}}, \tau\right)}{\theta_{1}\left(\frac{R_{t}^{\xi}}{4 \pi^{2}}, \tau\right)}\right)\right] d t .
\end{aligned}
$$

which is in $\Omega^{\text {odd }}(M, \mathbf{C})\left[\left[q^{\frac{1}{2}}\right]\right]$. Since $M$ is $4 k-1$ dimensional, $\left\{C S \Phi_{1}\left(\nabla^{T M}, \nabla^{V}, \nabla_{0}^{\xi}, \nabla_{1}^{\xi}, \tau\right)\right\}^{(4 k-1)}$ represents an element in $H^{4 k-1}(M, \mathbf{C})\left[\left[q^{\frac{1}{2}}\right]\right]$. Similarly, we can compute the transgressed forms for $\Phi_{2}$, and define 


$$
\begin{aligned}
& C S \Phi_{2}\left(\nabla^{T M}, \nabla^{V}, \nabla_{0}^{\xi}, \nabla_{1}^{\xi}, \tau\right) \\
& :=\frac{1}{8 \pi^{2}} \int_{0}^{1} \Phi_{2}\left(\nabla^{T M}, \nabla^{V}, \nabla_{t}^{\xi}, \tau\right) \operatorname{tr}\left[B\left(\frac{\theta_{3}^{\prime}\left(\frac{R_{t}^{\xi}}{4 \pi^{2}}, \tau\right)}{\theta_{3}\left(\frac{R_{t}^{\xi}}{4 \pi^{2}}, \tau\right)}+\frac{\theta_{1}^{\prime}\left(\frac{R_{t}^{\xi}}{4 \pi^{2}}, \tau\right)}{\theta_{1}\left(\frac{R_{t}^{\xi}}{4 \pi^{2}}, \tau\right)}-2 \frac{\theta_{2}^{\prime}\left(\frac{R_{t}^{\xi}}{4 \pi^{2}}, \tau\right)}{\theta_{2}\left(\frac{R_{t}^{\xi}}{4 \pi^{2}}, \tau\right)}\right)\right] d t
\end{aligned}
$$

which also lies in $\Omega^{\text {odd }}(M, \mathbf{C})\left[\left[q^{\frac{1}{2}}\right]\right]$ and its top component represents elements in $H^{4 k-1}(M, \mathbf{C})\left[\left[q^{\frac{1}{2}}\right]\right]$. Similar to Theorem 3.4 in $[\mathrm{W}]$, we have

Theorem 5.1 Let $M$ be a $4 k-1$ dimensional manifold and $\nabla^{T M}$ be a connection on $T M$ and $\xi$ be a two dimensional oriented Euclidean real vector bundle with two Euclidean connections $\nabla_{1}^{\xi}, \nabla_{0}^{\xi}$, then we have $\left\{C S \Phi_{1}\left(\nabla^{T M}, \nabla^{V}, \nabla_{0}^{\xi}, \nabla_{1}^{\xi}, \tau\right)\right\}^{(4 k-1)}$ is a modular form of weight $2 k$ over $\Gamma_{0}(2) ;\left\{C S \Phi_{2}\left(\nabla^{T M}, \nabla^{V}, \nabla_{0}^{\xi}, \nabla_{1}^{\xi}, \tau\right)\right\}^{(4 k-1)}$ is a modular form of weight $2 k$ over $\Gamma^{0}(2)$; The following equalities hold,

$$
\left\{C S \Phi_{1}\left(\nabla^{T M}, \nabla^{V}, \nabla_{0}^{\xi}, \nabla_{1}^{\xi}, \tau\right)\right\}^{(4 k-1)}=(2 \tau)^{2 k}\left\{C S \Phi_{2}\left(\nabla^{T M}, \nabla^{V}, \nabla_{0}^{\xi}, \nabla_{1}^{\xi}, \tau\right)\right\}^{(4 k-1)},
$$

Thus by Theorem 5.1, we can get a cancellation formula similar to Corollary 3.5 in $[\mathrm{W}]$.

Acknowledgement The work of the second author was supported by NSFC No.10801027 and Fok Ying Tong Education Foundation No. 121003.

\section{References}

[AW] L. Alvarez-Gaumé, E. Witten, Graviational anomalies, Nucl.Phys. B234 (1983), 269-330.

[C] K. Chandrasekharan, Elliptic Functions, Spinger-Verlag, 1985.

[CH1] Q. Chen, F. Han, Modular invariance and twisted anomaly cancellations of characteristic numbers, Trans. Amer. Math. Soc. 361 (2009), 1463-1493

[CH2] Q. Chen, F. Han, Elliptic genera, transgression and loop space Chern-Simons form, Comm. Anal. Geom., 17 (2009),:73-106.

[HLZ] F. Han, K. Liu, W. Zhang, Modular Forms and Generalized Anomaly Cancellation Formulas, arXiv:1109.2494.

[HZ1]F. Han, W. Zhang, Spin ${ }^{c}$-manifold and elliptic genera, C. R. Acad. Sci. Paris Serie I., 336 (2003), 1011-1014.

[HZ2] F. Han, W. Zhang, Modular invariance, characteristic numbers and eta Invariants, J. Diff. Geom. 67 (2004), 257-288.

[L] K. Liu, Modular invariance and characteristic numbers. Commu. Math. Phys. 174 (1995), 29-42.

[W] Y. Wang, Transgression and twisted anomaly cancellation formulas on odd dimensional manifolds, J. Geom. Phys.,60 (2010), 611-622.

[Z] W. Zhang, Lectures on Chern-weil Theory and Witten Deformations. Nankai Tracks in Mathematics Vol. 4, World Scientific, Singapore, 2001. 
Center of Mathematical Sciences, Zhejiang University Hangzhou Zhejiang 310027, China and Department of Mathematics, University of California at Los Angeles, Los Angeles CA 90095-1555, USA

Email: liu@ucla.edu.cn; liu@cms.zju.edu.cn

School of Mathematics and Statistics, Northeast Normal University, Changchun Jilin, 130024 China

E-mail: wangy581@nenu.edu.cn; 